\title{
Security Of Finite-Key-Length Measurement-Device-Independent Quantum Key Distribution Using Arbitrary Number Of Decoys
}

\author{
H. F. Chau* \\ Department of Physics, University of Hong Kong, Pokfulam Road, Hong Kong
}

(Dated: June 26, 2020)

\begin{abstract}
In quantum key distribution, measurement-device-independent and decoy-state techniques enable the two cooperative agents to establish a shared secret key using imperfect measurement devices and weak Poissonian sources, respectively. Investigations so far are not comprehensive as they restrict to less than or equal to four decoy states. Moreover, many of them involves pure numerical studies. Here I report a general security proof that works for any fixed number of decoy states and any fixed raw key length. The two key ideas involved here. The first one is the repeated application of the inversion formula for Vandermonde matrix to obtain various bounds on certain yields and error rates. The second one is the use of a recently proven generalization of the McDiarmid inequality. These techniques rise the best provably secure key rate of the measurement-device-independent version of the BB84 scheme by at least 1.25 times and increase the workable distance between the two cooperative agents from slightly less than $60 \mathrm{~km}$ to slightly greater than $130 \mathrm{~km}$ in case there are $10^{10}$ photon pulse pair sent without a quantum repeater.
\end{abstract}

\section{INTRODUCTION}

Quantum key distribution (QKD) is the art for two trusted agents, commonly refers to as Alice and Bob, to share a provably secure secret key by preparing and measuring quantum states that are transmitted through a noisy channel controlled by an eavesdropper Eve who has unlimited computational power. In realistic QKD setup, decoy-state technique allows Alice and Bob to obtain their secret key using the much more practical weak phase-randomized Poissonian sources [1, 2]. In addition, measurement-device-independent (MDI) method enables them to use imperfect apparatus that may be controlled by Eve to perform measurement [3]. Decoystate technique has been extensively studied. In fact, this technique can be applied to many different QKD schemes [1, 2, 4 6]. Researches on the effective use of a general number of decoys have been conducted [7-10]. The effect of finite raw key length on the key rate has been investigated [5, 8-11]. Nonetheless, security and efficiency analyses on the combined use of decoy-state and MDI techniques are less comprehensive. So far, they are restricted to less than or equal to four decoy states 12 21]. Furthermore, it is unclear how to extend these methods analytically to an arbitrary but fixed number of decoys. Along a slightly different line, the case of finite raw key length for the combined use of decoy-state and MDI techniques has been studied. So far, these studies applied Azuma, Hoeffding and Sefling inequalities as well as Chernoff bound in a straightforward manner [15, 1721.

Here I report the security analysis and a key rate formula for the BB84-based 22 MDI-QKD using passive partial Bell state detection for finite raw key length with the condition that Alice and Bob each uses an arbitrary

* email: hfchau@hku.hk but fixed number of decoys. One of the key ideas in this work is the repeated use of the analytical formula for the elements of the inverse of a Vandermonde matrix. A tight bound on various yields and error rates for a general number of decoys can then be obtained through this analytical formula. (Actually, Yuan et al. also used repeated Vandermonde matrix inversion to obtain upper and lower bounds of the so-called two-single-photon yield in case one of the photon intensities used is 0 [23]. Nevertheless, the bounds reported here are more general and powerful than theirs.) The other key idea used here is the application of a powerful generalization of the McDiarmid inequality in mathematical statistics recently proven in Ref. [10]. This inequality is effective to tackle finite size statistical fluctuation of certain error rates involved in the key rate formula.

I compute the secure key rate for the MDI-version of the BB84 scheme using the setup and channel studied by Zhou et al. in Ref. [18]. The best provably secure key rate for this setup before this work are reported by Mao et al. in Ref. [20]. Compared to their work, in case the total number of photon pulse pair send by Alice and Bob is $10^{10}$, the provably secure key rate using this new method is increased by at least $125 \%$. Besides, the maximum transmission distance is increased from slightly less than $60 \mathrm{~km}$ to slightly greater than $130 \mathrm{~km}$. This demonstrates the effectiveness of this new approach for MDI-QKD.

\section{THE MDI-QKD PROTOCOL}

In this paper, the polarization of all photon pulses are prepared either in X-basis with photon intensity $\mu_{\mathrm{X}, i}$ (for $\left.i=1,2, \cdots, k_{\mathrm{x}}\right)$ or in Z-basis with photon intensity $\mu_{\mathrm{z}, i}$ (for $i=1,2, \cdots, k_{\mathrm{z}}$ ). For simplicity, I label these photon intensities in descending order by $\mu_{\mathrm{x}, 1}>\mu_{\mathrm{x}, 2}>\cdots>$ $\mu_{\mathrm{x}, k_{\mathrm{x}}} \geq 0$ and similarly for $\mu_{\mathrm{z}, i}$ 's. I denote the probability of choosing the preparation basis $\mathrm{B} \in\{\mathrm{X}, \mathrm{Z}\}$ by $p_{\mathrm{B}}$ and the probability of choosing photon intensity $\mu_{\mathrm{B}, i}$ given 
the preparation basis B by $p_{i \mid \mathrm{B}}$.

Here I study the following MDI-QKD protocol, which is a BB84-based scheme originally studied in Refs. [3, 15].

1. Alice and Bob each has a phase-randomized Poissonian distributed source. Each of them randomly and independently prepares a photon pulse and sends it to the untrusted third party Charlie. They jot down the intensity and polarization used for each pulse.

2. Charlie performs a partial Bell state measurement like the ones in Refs. [3, 15, 24]. He publicly announces the measurement result including nondetection and inconclusive events.

3. Alice and Bob reveal the basis and intensity they used for each of their prepared photon pulse. If the preparation bases of a pair of photon pulses they have sent to Charlie for Bell basis measurement disagree, they discard them. If both pulses are prepared in the X-basis, they reveal their preparation polarizations. They also randomly reveal the preparation polarizations of a few pulses that they have both prepared in the Z-basis. In this way, they can estimate the various yields and error rates to be defined in Sec. III.

4. They use the preparation information of their remaining photon pulses that have been conclusively measured by Charlie to generate their raw secret keys and then perform error correction and privacy amplification on these keys to obtain their final secret keys according to the MDI-QKD procedure reported in Refs. [3, 24]. (Here I assume that Alice and Bob use forward reconciliation to establish the key. The case of reverse reconciliation can be studied in a similar manner.)

\section{BOUNDS ON VARIOUS YIELDS AND ERROR RATES IN THE MDI-SETTING}

I use the symbol $Q_{\mathrm{B}, i, j}$ to denote the yield given that both Alice and Bob prepare their photons in B-basis and that Alice (Bob) uses photon intensity $\mu_{\mathrm{B}, i}\left(\mu_{\mathrm{B}, j}\right)$ for $\mathrm{B}=\mathrm{X}, \mathrm{Z}$ and $i, j=1,2, \cdots, k_{\mathrm{B}}$. More precisely, it is the portion of photon pairs prepared using the above description that Charlie declares conclusive detection. Furthermore, I define the error rate of these photon pairs $E_{\mathrm{B}, i, j}$ as the portion of those conclusively detected photons above whose prepared polarizations by Alice and Bob are the same. And I set $\bar{E}_{\mathrm{B}, i, j}=1-E_{\mathrm{B}, i, j}$. Similar to the case of standard (that is, non-MDI) implementation of QKD, for phase randomized Poissonian photon sources [24],

$$
Q_{\mathrm{B}, i, j}=\sum_{a, b=0}^{+\infty} \frac{\mu_{\mathrm{B}, i}^{a} \mu_{\mathrm{B}, j}^{b} Y_{\mathrm{B}, a, b} \exp \left(-\mu_{\mathrm{B}, i}\right) \exp \left(-\mu_{\mathrm{B}, j}\right)}{a ! b !}
$$

and

$$
\begin{aligned}
& Q_{\mathrm{B}, i, j} E_{\mathrm{B}, i, j} \\
= & \sum_{a, b=0}^{+\infty} \frac{\mu_{\mathrm{B}, i}^{a} \mu_{\mathrm{B}, j}^{b} Y_{\mathrm{B}, a, b} e_{\mathrm{B}, a, b} \exp \left(-\mu_{\mathrm{B}, i}\right) \exp \left(-\mu_{\mathrm{B}, j}\right)}{a ! b !} .
\end{aligned}
$$

Here, $Y_{\mathrm{B}, a, b}$ is the probability of conclusive detection by Charlie given that the photon pulses sent by Alice (Bob) contains $a(b)$ photons and $e_{\mathrm{B}, a, b}$ is the corresponding bit error rate of the raw key. Furthermore, I denote the yield conditioned on Alice preparing a vacuum state and Bob preparing in the B-basis by the symbol $Y_{\mathrm{B}, 0, \star}$. Clearly, $Y_{\mathrm{B}, 0, \star}$ obeys

$$
Y_{\mathrm{B}, 0, \star}=\sum_{j=1}^{k_{\mathrm{B}}} p_{j \mid \mathrm{B}} \tilde{Y}_{\mathrm{B}, 0, j},
$$

where $\tilde{Y}_{\mathrm{B}, 0, j}$ is the yield conditioned on Alice sending the vacuum state and Bob sending photon with intensity $\mu_{\mathrm{B}, j}$ in the B-basis.

I need to deduce the possible values of $Y_{\mathrm{B}, i, j}$ 's and $Y_{\mathrm{B}, i, j} e_{\mathrm{B}, i, j}$ from Eqs. (11) and (2). One way to do it is to compute various lower and upper bounds of $Y_{\mathrm{B}, i, j}$ 's and $Y_{\mathrm{B}, i, j} e_{\mathrm{B}, i, j}$ by brute force optimization of truncated versions of Eqs. (11) and (2) like the method reported in Refs. [12, 15, 16]. However, this approach is rather inelegant and ineffective. Further note that Alice and Bob have no control on the values of $Y_{\mathrm{B}, a, b}$ 's and $e_{\mathrm{B}, a, b}$ 's since Charlie and Eve are not trustworthy. All they know is that these variables are between 0 and 1. Fortunately, in the case of phase-randomized Poissonian distributed light source, Corollaries 1 and 2 in the Appendix can be used to bound $Y_{\mathrm{B}, 0, \star}, Y_{\mathrm{B}, 1,1}, Y_{\mathrm{B}, 1,1} e_{\mathrm{B}, 1,1}$ and $Y_{\mathrm{B}, 1,1} \bar{e}_{\mathrm{B}, 1,1}$ analytically, where $\bar{e}_{\mathrm{B}, 1,1} \equiv 1-e_{\mathrm{B}, 1,1}$. More importantly, these bounds are effective to analyze the key rate formula to be reported in Sec. IV] Following the trick used in Refs. [9, 10], by using the statistics of either all the $k_{\mathrm{B}}$ different photon intensities or all but the largest one used by Alice and Bob depending on the parity of $k_{\mathrm{B}}$, Corollaries 1 and 2 imply the following tight bounds

$$
\begin{gathered}
Y_{\mathrm{B}, 0, \star} \geq \sum_{i, j=1}^{k_{\mathrm{B}}} p_{j \mid \mathrm{B}} \mathcal{A}_{\mathrm{B}, 0, i}^{\mathrm{e}} Q_{\mathrm{B}, i, j}, \\
Y_{\mathrm{B}, 1,1} \geq Y_{\mathrm{B}, 1,1}^{\downarrow} \equiv \sum_{i, j=1}^{k_{\mathrm{B}}} \mathcal{A}_{\mathrm{B}, 1, i}^{\mathrm{o}} \mathcal{A}_{\mathrm{B}, 1, j}^{\mathrm{o}} Q_{\mathrm{B}, i, j}-C_{\mathrm{B}, 2}^{2}, \\
Y_{\mathrm{B}, 1,1} e_{\mathrm{B}, 1,1} \leq\left(Y_{\mathrm{B}, 1,1} e_{\mathrm{B}, 1,1}\right)^{\uparrow} \equiv \sum_{i, j=1}^{k_{\mathrm{B}}} \mathcal{A}_{\mathrm{B}, 1, i}^{\mathrm{e}} \mathcal{A}_{\mathrm{B}, 1, j}^{\mathrm{e}} Q_{\mathrm{B}, i, j} E_{\mathrm{B}, i, j}, \\
Y_{\mathrm{B}, 1,1} e_{\mathrm{B}, 1,1} \geq\left(Y_{\mathrm{B}, 1,1} e_{\mathrm{B}, 1,1}\right)^{\downarrow} \\
\equiv \sum_{i, j=1}^{k_{\mathrm{B}}} \mathcal{A}_{\mathrm{B}, 1, i}^{\mathrm{o}} \mathcal{A}_{\mathrm{B}, 1, j}^{\mathrm{o}} Q_{\mathrm{B}, i, j} E_{\mathrm{B}, i, j}-C_{\mathrm{B}, 2}^{2},
\end{gathered}
$$


and

$$
\begin{aligned}
Y_{\mathrm{B}, 1,1} \bar{e}_{\mathrm{B}, 1,1} & \geq\left(Y_{\mathrm{B}, 1,1} \bar{e}_{\mathrm{B}, 1,1}\right)^{\downarrow} \\
& \equiv \sum_{i, j=1}^{k_{\mathrm{B}}} \mathcal{A}_{\mathrm{B}, 1, i}^{\mathrm{o}} \mathcal{A}_{\mathrm{B}, 1, j}^{\mathrm{o}} Q_{\mathrm{B}, i, j} \bar{E}_{\mathrm{B}, i, j}-C_{\mathrm{B}, 2}^{2}
\end{aligned}
$$

for $\mathrm{B}=\mathrm{X}, \mathrm{Z}$. (The reason for using e and o as superscripts is that it will be self-evident from the discussion below that for fixed $\mathrm{B}$ and $j$, there are even number of nonzero terms in $\mathcal{A}_{\mathrm{B}, j, i}^{\mathrm{e}}$ and odd number of non-zero terms in $\mathcal{A}_{\mathrm{B}, j, i}^{\mathrm{o}}$.) For the above inequalities, in case $k_{\mathrm{B}}$ is even, then

$$
\mathcal{A}_{\mathrm{B}, j, i}^{\mathrm{e}}=A_{j}\left(\mu_{\mathrm{B}, i},\left\{\mu_{\mathrm{B}, 1}, \mu_{\mathrm{B}, 2}, \cdots, \mu_{\mathrm{B}, i-1}, \mu_{\mathrm{B}, i+1}, \cdots, \mu_{\mathrm{B}, k_{\mathrm{B}}}\right\}\right)
$$

for $i=1,2, \cdots, k_{\mathrm{B}}$ and $j=0,1$. Furthermore,

$$
\mathcal{A}_{\mathrm{B}, 1,1}^{\mathrm{O}}=0
$$

and

$$
\mathcal{A}_{\mathrm{B}, 1, i}^{\mathrm{O}}=A_{1}\left(\mu_{\mathrm{B}, i},\left\{\mu_{\mathrm{B}, 2}, \mu_{\mathrm{B}, 3}, \cdots, \mu_{\mathrm{B}, i-1}, \mu_{\mathrm{B}, i+1}, \cdots, \mu_{\mathrm{B}, k_{\mathrm{B}}}\right\}\right)
$$

for $i=2,3, \cdots, k_{\mathrm{B}}$. In addition,

$$
C_{\mathrm{B}, 2}=\left(\sum_{\ell=2}^{k_{\mathrm{B}}} \mu_{\mathrm{B}, 2} \mu_{\mathrm{B}, 3} \cdots \mu_{\mathrm{B}, \ell-1} \mu_{\mathrm{B}, \ell+1} \cdots \mu_{\mathrm{B}, k_{\mathrm{B}}}\right) \sum_{i=2}^{k_{\mathrm{B}}}\left\{\frac{1}{\mu_{\mathrm{B}, i} \prod_{t \neq 1, i}\left(\mu_{\mathrm{B}, i}-\mu_{\mathrm{B}, t}\right)}\left[\exp \left(\mu_{\mathrm{B}, i}\right)-\sum_{j=0}^{k_{\mathrm{B}}-2} \frac{\mu_{\mathrm{B}, i}^{j}}{j !}\right]\right\} .
$$

Here I use the convention that the term involving $1 / \mu_{\mathrm{B}, i}$ in the above summards with dummy index $i$ is equal to 0 if $\mu_{\mathrm{B}, i}=0$.

Whereas in case $k_{\mathrm{B}}$ is odd, then

$\mathcal{A}_{\mathrm{B}, 1, i}^{\mathrm{o}}=A_{1}\left(\mu_{\mathrm{B}, i},\left\{\mu_{\mathrm{B}, 1}, \mu_{\mathrm{B}, 2}, \cdots, \mu_{\mathrm{B}, i-1}, \mu_{\mathrm{B}, i+1}, \cdots, \mu_{\mathrm{B}, k_{\mathrm{B}}}\right\}\right)$ for $i=1,2, \cdots, k_{\mathrm{B}}$. Furthermore,

$$
\mathcal{A}_{\mathrm{B}, j, 1}^{\mathrm{e}}=0
$$

and

$\mathcal{A}_{\mathrm{B}, j, i}^{\mathrm{e}}=A_{j}\left(\mu_{\mathrm{B}, i},\left\{\mu_{\mathrm{B}, 2}, \mu_{\mathrm{B}, 3}, \cdots, \mu_{\mathrm{B}, i-1}, \mu_{\mathrm{B}, i+1}, \cdots, \mu_{\mathrm{B}, k_{\mathrm{B}}}\right\}\right)$

for $j=1,2$ and $i=2,3, \cdots, k_{\mathrm{B}}$. In addition,
Note that in Eq. (5),

$$
A_{0}(\mu, S)=\frac{-\exp (\mu) \prod_{s \in S} s}{\prod_{s \in S}(\mu-s)}
$$

and

$$
A_{1}(\mu, S)=\frac{-\exp (\mu) \sum_{s \in S}\left(\prod_{\substack{s^{\prime} \in S \\ s^{\prime} \neq s}} s^{\prime}\right.}{\prod_{s \in S}(\mu-s)} .
$$

Note that different upper and lower bounds for $Y_{\mathrm{B}, 1,1}$ have been obtained using similar Vandermonde matrix inversion technique in Ref. [23]. The differences between those bounds and the actual value of $Y_{\mathrm{B}, 1,1}$ depend on the yields $Y_{\mathrm{B}, i, j}$ with $i, j \geq 1$. In contrast, the difference between the bound in Inequalities (4b) and the actual value of $Y_{\mathrm{B}, 1,1}$ depend on $Y_{\mathrm{B}, i, j}$ with $i, j \geq k_{\mathrm{B}}$. Thus, Inequality (4b) and similarly also Inequality (4c) give more accurate estimates of $Y_{\mathrm{B}, 1,1}$ and $Y_{\mathrm{B}, 1,1} e_{\mathrm{B}, 1,1}$, respectively. Furthermore, the bounds in Ref. 23 also work for the case of $\mu_{\mathrm{B}, k_{\mathrm{B}}}=0$. It is also not clear how to extend their method to bound for yields other than the two-singlephoton events that are needed in computing the key rate for twin-field [25] and phase-matching [26] MDI-QKDs.

\section{THE KEY RATE FORMULA}

The secure key rate $R$ is defined as the number of bits of secret key shared by Alice and Bob at the end of the protocol divided by the number of photon pulse pairs they have sent to Charlie. In fact, the derivation of the key rate formula in Refs. [9-11, 27] for the case of standard QKD can be easily modified to the case of MDI- 
QKD by making the following correspondences. (See also the key rate formula used in Ref. 24] for MDI-QKD.) The vacuum event in the standard QKD is mapped to the event that both Alice and Bob send a vacuum photon pulse to Charlie. The single photon event is mapped to the event that both Alice and Bob send a single photon to Charlie. The multiple photon event is mapped to the event that Alice and Bob are both sending neither a vacuum nor a single photon pulse to Charlie. In the case of forward reconciliation, the result is

$$
R \geq p_{\mathrm{z}}^{2}\left\{\langle\exp (-\mu)\rangle_{\mathrm{z}} Y_{\mathrm{Z}, 0, \star}+\langle\mu \exp (-\mu)\rangle_{\mathrm{z}}^{2} Y_{\mathrm{z}, 1,1}\left[1-H_{2}\left(e_{p}\right)\right]-\Lambda_{\mathrm{EC}}-\frac{\left\langle Q_{\mathrm{z}, i, j}\right\rangle_{i, j}}{\ell_{\mathrm{raw}}}\left[6 \log _{2}\left(\frac{\chi}{\epsilon_{\mathrm{sec}}}\right)+\log _{2}\left(\frac{2}{\epsilon_{\mathrm{cor}}}\right)\right]\right\}
$$

where $\langle f(\mu)\rangle_{\mathrm{z}} \equiv \sum_{i=1}^{k_{\mathrm{z}}} p_{i \mid \mathrm{Z}} f\left(\mu_{\mathrm{Z}, i}\right), \quad\langle f(\mathrm{Z}, i, j)\rangle_{i, j} \equiv$ $\sum_{i, j=1}^{k_{\mathrm{Z}}} p_{i \mid \mathrm{Z}} p_{j \mid \mathrm{Z}} f(\mathrm{Z}, i, j), \quad H_{2}(x) \equiv-x \log _{2} x-(1-$ $x) \log _{2}(1-x)$ is the binary entropy function, $e_{p}$ is the phase error rate of the single photon events in the raw key, and $\Lambda_{\mathrm{EC}}$ is the actual number of bits of information that leaks to Eve as Alice and Bob perform error correction on their raw bits. It is given by

$$
\Lambda_{\mathrm{EC}}=f_{\mathrm{EC}}\left\langle Q_{\mathrm{z}, i, j} H_{2}\left(E_{\mathrm{z}, i, j}\right)\right\rangle_{i, j}
$$

where $f_{\mathrm{EC}} \geq 1$ measures the inefficiency of the errorcorrecting code used. In addition, $\ell_{\text {raw }}$ is the raw sifted key length measured in bits, $\epsilon_{\mathrm{cor}}$ is the upper bound of the probability that the final keys shared between Alice and Bob are different, and $\epsilon_{\mathrm{sec}}=\left(1-p_{\text {abort }}\right) \| \rho_{\mathrm{AE}}-U_{\mathrm{A}} \otimes$ $\rho_{\mathrm{E}} \|_{1} / 2$. Here $p_{\text {abort }}$ is the chance that the scheme aborts without generating a key, $\rho_{\mathrm{AE}}$ is the classical-quantum state describing the joint state of Alice and Eve, $U_{\mathrm{A}}$ is the uniform mixture of all the possible raw keys created by Alice, $\rho_{\mathrm{E}}$ is the reduced density matrix of Eve, and $\|\cdot\|_{1}$ is the trace norm [28 30]. In other words, Eve has at most $\epsilon_{\mathrm{sec}}$ bits of information on the final secret key shared by Alice and Bob. (In the literature, this is often referred to it as a $\epsilon_{\text {cor-correct }}$ and $\epsilon_{\text {sec-secure }}$ QKD scheme [27].) Last but not least, $\chi$ is a QKD scheme specific factor which depends on the detailed security analysis used. In general, $\chi$ may also depend on other factors used in the QKD scheme such as the number of photon intensities $k_{\mathrm{X}}$ and $k_{\mathrm{Z}}[9-11]$.

In Inequality (7), the phase error of the raw key $e_{p}$ obeys 9 , 31]

$$
e_{p} \leq e_{\mathrm{X}, 1,1}+\bar{\gamma}\left(\frac{\epsilon_{\mathrm{sec}}}{\chi}, e_{\mathrm{X}, 1,1}, \frac{s_{\mathrm{X}} Y_{\mathrm{X}, 1,1}\langle\mu \exp (-\mu)\rangle_{\mathrm{X}}^{2}}{\left\langle Q_{\mathrm{X}, i, j}\right\rangle_{i, j}}, \frac{s_{\mathrm{Z}} Y_{\mathrm{Z}, 1,1}\langle\mu \exp (-\mu)\rangle_{\mathrm{Z}}^{2}}{\left\langle Q_{\mathrm{Z}, i, j}\right\rangle_{i, j}}\right)
$$

with probability at least $1-\epsilon_{\mathrm{sec}} / \chi$, where $\langle f(\mu)\rangle_{\mathrm{x}} \equiv$ $\sum_{i=1}^{k_{\mathrm{x}}} p_{i \mid \mathrm{X}} f\left(\mu_{\mathrm{x}, i}\right)$,

$$
\bar{\gamma}(a, b, c, d) \equiv \sqrt{\frac{(c+d)(1-b) b}{c d} \ln \left[\frac{c+d}{2 \pi c d(1-b) b a^{2}}\right]}
$$

and $s_{\mathrm{B}}$ is the number of bits that are prepared and measured in B basis. Clearly, $s_{\mathrm{Z}}=\ell_{\text {raw }}$ and $s_{\mathrm{X}} \approx$ $p_{\mathrm{X}}^{2} s_{\mathrm{Z}}\left\langle Q_{\mathrm{X}, i, j}\right\rangle_{i, j} /\left(p_{\mathrm{Z}}^{2}\left\langle Q_{\mathrm{Z}, i, j}\right\rangle_{i, j}\right)$. I also remark that $\bar{\gamma}$ becomes complex if $a, c, d$ are too large. This is because in this case no $e_{p} \geq e_{\mathrm{X}, 1,1}$ exists with failure probability $a$. In this work, all parameters are carefully picked so that $\bar{\gamma}$ is real.

There are two ways to proceed. The most general way is to directly find a lower bound for $Y_{\mathrm{z}, 1,1}$. Specifically, by substituting Inequalities (4a), (4b) and (9) into Inequality (7), I obtain the following lower bound of the key rate

$$
\begin{aligned}
R \geq & \sum_{i, j=1}^{k_{\mathrm{z}}} \mathcal{B}_{\mathrm{z}, i, j} Q_{\mathrm{z}, i, j}-p_{\mathrm{z}}^{2}\left\{\langle\mu \exp (-\mu)\rangle_{\mathrm{z}}^{2} C_{\mathrm{z}, 2}^{2}\left[1-H_{2}\left(e_{p}\right)\right]\right. \\
& \left.+\Lambda_{\mathrm{EC}}+\frac{\left\langle Q_{\mathrm{z}, i, j}\right\rangle_{i, j}}{\ell_{\text {raw }}}\left[6 \log _{2}\left(\frac{\chi}{\epsilon_{\mathrm{sec}}}\right)+\log _{2}\left(\frac{2}{\epsilon_{\mathrm{cor}}}\right)\right]\right\},
\end{aligned}
$$

where

$$
\begin{aligned}
\mathcal{B}_{\mathrm{Z}, i, j}= & p_{\mathrm{Z}}^{2}\left\{\langle\exp (-\mu)\rangle_{\mathrm{Z}} \mathcal{A}_{\mathrm{z}, 0, i}^{\mathrm{e}} p_{j \mid \mathrm{Z}}\right. \\
& \left.+\langle\mu \exp (-\mu)\rangle_{\mathrm{z}}^{2} \mathcal{A}_{\mathrm{z}, 1, i}^{\mathrm{o}} \mathcal{A}_{\mathrm{z}, 1, j}^{\mathrm{o}}\left[1-H_{2}\left(e_{p}\right)\right]\right\} .
\end{aligned}
$$

Here I would like to point out that unlike the corresponding key rate formulae for standard QKD in Refs. 7, 9 11], a distinctive feature of the key rate formula for MDI-QKD in Eq. (11) is the presence of the $C_{\mathrm{z}, 2}^{2}$ term. From Eq. (5), provided that $\mu_{\mathrm{Z}, i}-\mu_{\mathrm{Z}, i+1}$ are all greater than a fixed positive number, the value of $C_{\mathrm{z}, 2}^{2}$ decreases with $k_{\mathrm{Z}}$. This is the reason why the MDI version of a QKD scheme may require more decoys to attain a 
key rate comparable to the corresponding standard QKD scheme.

There is an alternative way to obtain the key rate formula discovered by Zhou et al. [18] that works for BB84 [22] and the six-state scheme [32]. Suppose the photon pulses prepared by Alice and Bob in Step 1 of the MDI-QKD protocol in Sec. II both contain a single photon. Suppose further that they are prepared in the same basis. Then, from Charlie and Eve's point of view, this two-single-photon state are the same irrespective of their preparation basis. Consequently, $Y_{\mathrm{X}, 1,1}=Y_{\mathrm{Z}, 1,1}$ (even though $e_{\mathrm{X}, 1,1}$ need not equal $\left.e_{\mathrm{Z}, 1,1}\right)$. That is to say, the secure key rate in Inequality (7) also holds if $Y_{\mathrm{Z}, 1,1}$ there is replaced by $Y_{\mathrm{X}, 1,1}$. (Here I stress that the key generation basis is still $\mathrm{Z}$. But as $Y_{\mathrm{X}, 1,1}=Y_{\mathrm{Z}, 1,1}$, I could use the bound on $Y_{\mathrm{X}, 1,1}$ to obtain an alternative key rate formula for the same MDI-QKD scheme.) Following the same procedure above, I get

$$
\begin{aligned}
R \geq & \sum_{i, j=1}^{k_{\mathrm{z}}} \mathcal{B}_{\mathrm{z}, i, j}^{\prime} Q_{\mathrm{z}, i, j}+\sum_{i, j=1}^{k_{\mathrm{x}}} \mathcal{B}_{\mathrm{x}, i, j} Q_{\mathrm{x}, i, j} \\
& -p_{\mathrm{Z}}^{2}\left\{\langle\mu \exp (-\mu)\rangle_{\mathrm{Z}}^{2} C_{\mathrm{x}, 2}^{2}\left[1-H_{2}\left(e_{p}\right)\right]+\Lambda_{\mathrm{EC}}\right. \\
& \left.+\frac{\left\langle Q_{\mathrm{z}, i, j}\right\rangle_{i, j}}{\ell_{\text {raw }}}\left[6 \log _{2}\left(\frac{\chi}{\epsilon_{\mathrm{sec}}}\right)+\log _{2}\left(\frac{2}{\epsilon_{\mathrm{cor}}}\right)\right]\right\},
\end{aligned}
$$

where

$$
\mathcal{B}_{\mathrm{Z}, i, j}^{\prime}=p_{\mathrm{Z}}^{2}\langle\exp (-\mu)\rangle_{\mathrm{Z}} \mathcal{A}_{\mathrm{Z}, 0, i}^{\mathrm{e}} p_{j \mid \mathrm{Z}}
$$

and

$$
\mathcal{B}_{\mathrm{X}, i, j}=p_{\mathrm{Z}}^{2}\langle\mu \exp (-\mu)\rangle_{\mathrm{Z}}^{2} \mathcal{A}_{\mathrm{x}, 1, i}^{\mathrm{o}} \mathcal{A}_{\mathrm{x}, 1, j}^{\mathrm{o}}\left[1-H_{2}\left(e_{p}\right)\right]
$$

\section{TREATMENTS OF PHASE ERROR AND STATISTICAL FLUCTUATION DUE TO FINITE RAW KEY LENGTH ON THE SECURE KEY RATE}

In order to compute the lower bound on the key rate $R$ in Inequalities (11) and (13), I need to know the value of $e_{\mathrm{X}, 1,1}$ through the Inequality (9). More importantly, I need to take into consideration the effects of finite raw key length on the key rate $R$ due to the statistical fluctuations in $e_{\mathrm{x}, 1,1}$ and $Q_{\mathrm{z}, i, j}$ 's. Here I do so by means of a McDiarmid-type of inequality in statistics first proven in Refs. [33, 34] and recently extended in Ref. [10].

Fluctuation of the first term in the R.H.S. of Inequality (11) due to finite raw key length can be handled by Hoeffding inequality for hypergeometrically distributed random variables $9-11,35]$, which is a special case of the McDiarmid inequality. Using the technique reported in Refs. [9, 10], the first term in the R.H.S. of Inequality (11) can be regarded as a sum of $s_{z}$ hypergeometrically distributed random variables each taking on values from the set $\left\{\left\langle Q_{\mathrm{Z}, i, j}\right\rangle_{i, j} \mathcal{B}_{\mathrm{Z}, i, j} /\left(p_{i \mid \mathrm{z}} p_{j \mid \mathrm{Z}}\right)\right\}_{i, j=1}^{k_{\mathrm{Z}}}$. Using Hoeffding inequality for hypergeometrically distributed random variables [35], I conclude that the measured value of $\sum_{i, j} \mathcal{B}_{i j} Q_{\mathrm{z}, i, j}$ minus its actual value is greater than $\left\langle Q_{\mathrm{Z}, i, j}\right\rangle_{i, j}\left[\frac{\ln \left(\chi / \epsilon_{\mathrm{sec}}\right)}{2 s_{\mathrm{Z}}}\right]^{1 / 2}$ Width $\left(\left\{\frac{\mathcal{B}_{\mathrm{Z}, i, j}}{p_{i \mid \mathrm{z}} p_{j \mid \mathrm{z}}}\right\}_{i, j=1}^{k_{\mathrm{z}}}\right) \quad$ with probability at most $\epsilon_{\mathrm{sec}} / \chi$, where Width of a finite set of real numbers $S$ is defined as $\max S-\min S$.

The value of $e_{\mathrm{x}, 1,1}$ in the finite sampling size situation is more involved. Here I adapt the recent results in Ref. [10. to give four upper bounds on $e_{\mathrm{X}, 1,1}$. Surely, I pick the best upper bound out of these four in the key rate analysis. The first step is to use the equality

$$
\begin{aligned}
e_{\mathrm{X}, 1,1} & =\frac{Y_{\mathrm{X}, 1,1} e_{\mathrm{X}, 1,1}}{Y_{\mathrm{X}, 1,1}} \\
& =\frac{Y_{\mathrm{X}, 1,1} e_{\mathrm{X}, 1,1}}{Y_{\mathrm{X}, 1,1} e_{\mathrm{X}, 1,1}+Y_{\mathrm{X}, 1,1} \bar{e}_{\mathrm{X}, 1,1}} .
\end{aligned}
$$

To get the first two upper bounds of $e_{\mathrm{X}, 1,1}$, I follow Ref. [10] by using Inequalities (4b), (4c) and (4e) together with applying Hoeffding inequality for hypergeometrically distributed random variables to study the statistical fluctuations of $\sum_{i, j=1}^{k_{\mathrm{x}}} \mathcal{A}_{\mathrm{x}, 1, i}^{\mathrm{e}} \mathcal{A}_{\mathrm{x}, 1, j}^{\mathrm{e}} Q_{\mathrm{x}, i, j} E_{\mathrm{x}, i, j}, \quad \sum_{i, j=1}^{k_{\mathrm{x}}} \mathcal{A}_{\mathrm{x}, 1, i}^{\mathrm{o}} \mathcal{A}_{\mathrm{x}, 1, j}^{\mathrm{o}} Q_{\mathrm{x}, i, j}$ and $\sum_{i, j=1}^{k_{\mathrm{x}}} \mathcal{A}_{\mathrm{x}, 1, i}^{\mathrm{o}} \mathcal{A}_{\mathrm{x}, 1, j}^{\mathrm{o}} Q_{\mathrm{x}, i, j} \bar{E}_{\mathrm{X}, i, j}$. The result is

$$
e_{\mathrm{X}, 1,1} \leq \frac{\left(Y_{\mathrm{X}, 1,1} e_{\mathrm{X}, 1,1}\right)^{\uparrow}+\Delta Y_{\mathrm{X}, 1,1} e_{\mathrm{X}, 1,1}}{Y_{\mathrm{X}, 1,1}^{\downarrow}-\Delta Y_{\mathrm{X}, 1,1}}
$$

and

$$
\begin{aligned}
e_{\mathrm{X}, 1,1} \leq & {\left[\left(Y_{\mathrm{X}, 1,1} e_{\mathrm{X}, 1,1}\right)^{\uparrow}+\Delta Y_{\mathrm{X}, 1,1} e_{\mathrm{X}, 1,1}\right]\left[\left(Y_{\mathrm{X}, 1,1} e_{\mathrm{X}, 1,1}\right)^{\uparrow}\right.} \\
& \left.+\left(Y_{\mathrm{X}, 1,1} \bar{e}_{\mathrm{X}, 1,1}\right)^{\downarrow}+\Delta Y_{\mathrm{X}, 1,1} e_{\mathrm{X}, 1,1}-\Delta Y_{\mathrm{X}, 1,1} \bar{e}_{\mathrm{X}, 1,1}\right]^{-1}
\end{aligned}
$$

each with probability at least $1-2 \epsilon_{\mathrm{sec}} / \chi$, where

$$
\begin{gathered}
\Delta Y_{\mathrm{X}, 1,1} e_{\mathrm{X}, 1,1}=\left[\frac{\left\langle Q_{\mathrm{x}, i, j}\right\rangle_{i, j}\left\langle Q_{\mathrm{x}, i, j} E_{\mathrm{X}, i, j}\right\rangle_{i, j} \ln \left(\chi / \epsilon_{\mathrm{sec}}\right)}{2 s_{\mathrm{X}}}\right]^{1 / 2} \times \\
\operatorname{Width}\left(\left\{\frac{\mathcal{A}_{\mathrm{x}, 1, i}^{\mathrm{e}} \mathcal{A}_{\mathrm{x}, 1, j}^{\mathrm{e}}}{p_{i \mid \mathrm{x}} p_{j \mid \mathrm{x}}}\right\}_{i, j=1}^{k_{\mathrm{x}}}\right),(18 \mathrm{a}) \\
\Delta Y_{\mathrm{X}, 1,1}=\left\langle Q_{\mathrm{x}, i, j}\right\rangle_{i, j}\left[\frac{\ln \left(\chi / \epsilon_{\mathrm{sec}}\right)}{2 s_{\mathrm{X}}}\right]^{1 / 2} \times \\
\operatorname{Width}\left(\left\{\frac{\mathcal{A}_{\mathrm{x}, 1, i}^{\mathrm{o}} \mathcal{A}_{\mathrm{x}, 1, j}^{\mathrm{o}}}{p_{i \mid \mathrm{x}} p_{j \mid \mathrm{x}}}\right\}_{i, j=1}^{k_{\mathrm{x}}}\right)
\end{gathered}
$$

and

$$
\begin{aligned}
\Delta Y_{\mathrm{X}, 1,1} \bar{e}_{\mathrm{X}, 1,1}= & {\left[\frac{\left\langle Q_{\mathrm{X}, i, j}\right\rangle_{i, j}\left\langle Q_{\mathrm{x}, i, j} \bar{E}_{\mathrm{X}, i, j}\right\rangle_{i, j} \ln \left(\chi / \epsilon_{\mathrm{sec}}\right)}{2 s_{\mathrm{X}}}\right]^{1 / 2} \times } \\
& \operatorname{Width}\left(\left\{\frac{\mathcal{A}_{\mathrm{X}, 1, i}^{\mathrm{O}} \mathcal{A}_{\mathrm{x}, 1, j}^{\mathrm{O}}}{p_{i \mid \mathrm{x}} p_{j \mid \mathrm{X}}}\right\}_{i, j=1}^{k_{\mathrm{X}}}\right) \cdot(18 \mathrm{c})
\end{aligned}
$$


Note that in the above equations, $\langle f(\mathrm{X}, i, j)\rangle_{i, j} \equiv$ inequality in Ref. [10]. For the third one, the result is $\sum_{i, j=1}^{k_{\mathrm{x}}} p_{i \mid \mathrm{x}} p_{j \mid \mathrm{X}} f(\mathrm{x}, i, j)$.

$$
\begin{aligned}
e_{\mathrm{X}, 1,1} \leq & \frac{\left(Y_{\mathrm{X}, 1,1} e_{\mathrm{X}, 1,1}\right)^{\uparrow}}{\left(Y_{\mathrm{X}, 1,1} e_{\mathrm{X}, 1,1}\right)^{\uparrow}+\left(Y_{\mathrm{X}, 1,1} \bar{e}_{\mathrm{X}, 1,1}\right)^{\downarrow}-\Delta Y_{\mathrm{X}, 1,1} \bar{e}_{\mathrm{X}, 1,1}} \\
& +\Delta e_{\mathrm{X}, 1,1}
\end{aligned}
$$

Both the third and the fourth bounds of $e_{\mathrm{X}, 1,1}$ use Eq. (15b), Inequality (4e) and the modified McDiarmid

with probability at least $1-2 \epsilon_{\mathrm{sec}} / \chi$, where

$$
\begin{aligned}
& \Delta e_{\mathrm{x}, 1,1} \\
& =\left[\frac{\left\langle Q_{\mathrm{x}, i, j}\right\rangle_{i, j}\left\langle Q_{\mathrm{x}, i, j} E_{\mathrm{X}, i, j}\right\rangle_{i, j} \ln \left(\chi / \epsilon_{\mathrm{sec}}\right)}{2 s_{\mathrm{X}}}\right]^{1 / 2}\left[\left(Y_{\mathrm{X}, 1,1} \bar{e}_{\mathrm{X}, 1,1}\right)^{\downarrow}-\Delta Y_{\mathrm{X}, 1,1} \bar{e}_{\mathrm{X}, 1,1}\right] \operatorname{Width}\left(\left\{\frac{\mathcal{A}_{\mathrm{x}, 1, i}^{\mathrm{e}} \mathcal{A}_{\mathrm{x}, 1, j}^{\mathrm{e}}}{p_{i \mid \mathrm{x}} p_{j \mid \mathrm{X}}}\right\}_{i, j=1}^{k_{\mathrm{x}}}\right) \\
& \times\left[\left(Y_{\mathrm{X}, 1,1} \bar{e}_{\mathrm{X}, 1,1}\right)^{\downarrow}-\Delta Y_{\mathrm{X}, 1,1} \bar{e}_{\mathrm{X}, 1,1}+\left(Y_{\mathrm{X}, 1,1} e_{\mathrm{X}, 1,1}\right)^{\downarrow}\left(1-\frac{\left\langle Q_{\mathrm{x}, i, j}\right\rangle_{i, j}}{s_{\mathrm{X}}\left\langle Q_{\mathrm{x}, i, j} E_{\mathrm{X}, i, j}\right\rangle_{i, j}}\right)+\frac{\left\langle Q_{\mathrm{x}, i, j}\right\rangle_{i, j}^{2}}{s_{\mathrm{X}}^{2}\left\langle Q_{\mathrm{X}, i, j} E_{\mathrm{X}, i, j}\right\rangle_{i, j}} \operatorname{maxx}_{i, j=1}^{k_{\mathrm{X}}}\left\{\frac{\mathcal{A}_{\mathrm{X}, 1, i}^{\mathrm{e}} \mathcal{A}_{\mathrm{x}, 1, j}^{\mathrm{e}}}{p_{i \mid \mathrm{x}} p_{j \mid \mathrm{X}}}\right\}\right]^{-1} \\
& \times\left[\left(Y_{\mathrm{x}, 1,1} \bar{e}_{\mathrm{X}, 1,1}\right)^{\downarrow}-\Delta Y_{\mathrm{x}, 1,1} \bar{e}_{\mathrm{X}, 1,1}+\left(Y_{\mathrm{x}, 1,1} e_{\mathrm{X}, 1,1}\right)^{\downarrow}\left(1-\frac{\left\langle Q_{\mathrm{x}, i, j}\right\rangle_{i, j}}{s_{\mathrm{X}}\left\langle Q_{\mathrm{x}, i, j} E_{\mathrm{X}, i, j}\right\rangle_{i, j}}\right)+\frac{\left\langle Q_{\mathrm{x}, i, j}\right\rangle_{i, j}^{2}}{s_{\mathrm{X}}^{2}\left\langle Q_{\mathrm{x}, i, j} E_{\mathrm{X}, i, j}\right\rangle_{i, j}} \min _{i, j=1}\left\{\frac{\mathcal{A}_{\mathrm{x}, 1, i} \mathcal{A}_{\mathrm{X}, 1, j}^{\mathrm{e}}}{p_{i \mid \mathrm{x}} p_{j \mid \mathrm{x}}}\right\}\right]^{-1} .
\end{aligned}
$$

And the fourth bound is

$$
e_{\mathrm{X}, 1,1} \leq \frac{\left(Y_{\mathrm{X}, 1,1} e_{\mathrm{X}, 1,1}\right)^{\uparrow}}{\left(Y_{\mathrm{X}, 1,1} e_{\mathrm{X}, 1,1}\right)^{\uparrow}+\left(Y_{\mathrm{X}, 1,1} \bar{e}_{\mathrm{X}, 1,1}\right)^{\downarrow}-\Delta Y_{\mathrm{X}, 1,1} \bar{e}_{\mathrm{X}, 1,1}}+\hat{r}\left[\frac{\ln \left(\chi / \epsilon_{\mathrm{sec}}\right)}{2}\right]^{1 / 2}
$$

with probability at least $1-3 \epsilon_{\mathrm{sec}} / \chi$, where

$$
\begin{aligned}
\hat{r}^{2} \approx y^{2} & \sum_{m=1}^{k_{\mathrm{x}}^{2}} \frac{1}{w^{(m)}-x}\left(-\frac{1}{y+\left(t-\sum_{i<m} n^{(i)}+1\right) x+\min \mathcal{W}+\sum_{i<m} n^{(i)} w^{(i)}+\mu\left[w^{(m)}-x\right]}\right. \\
& -\frac{1}{y+\left(t-\sum_{i<m} n^{(i)}+1\right) x+\max \mathcal{W}+\sum_{i<m} n^{(i)} w^{(i)}+\mu\left[w^{(m)}-x\right]} \\
& \left.+\frac{2}{\operatorname{Width}(\mathcal{W})} \ln \left\{\frac{y+\left(t-\sum_{i<m} n^{(i)}+1\right) x+\max \mathcal{W}+\sum_{i<m} n^{(i)} w^{(i)}+\mu\left[w^{(m)}-x\right]}{y+\left(t-\sum_{i<m} n^{(i)}+1\right) x+\min \mathcal{W}+\sum_{i<m} n^{(i)} w^{(i)}+\mu\left[w^{(m)}-x\right]}\right\}\right)\left.\right|_{\mu=0} ^{n^{(m)}} .
\end{aligned}
$$

In the above equation,

$$
\begin{gathered}
y=\left(Y_{\mathrm{X}, 1,1} \bar{e}_{\mathrm{X}, 1,1}\right)^{\downarrow}-\Delta Y_{\mathrm{X}, 1,1} \bar{e}_{\mathrm{X}, 1,1}, \\
t \approx \frac{s_{\mathrm{X}}\left\langle Q_{\mathrm{X}, i, j} E_{\mathrm{X}, i, j}\right\rangle_{i, j}}{\left\langle Q_{\mathrm{X}, i, j}\right\rangle_{i, j}}, \\
x=\frac{\left(Y_{\mathrm{X}, 1,1} e_{\mathrm{X}, 1,1}\right)^{\downarrow}-\Delta Y_{\mathrm{X}, 1,1} e_{\mathrm{X}, 1,1}}{t}
\end{gathered}
$$

and

$$
\mathcal{W}=\left\{\frac{\left\langle Q_{\mathrm{x}, i^{\prime}, j^{\prime}}\right\rangle_{i^{\prime}, j^{\prime}} \mathcal{A}_{\mathrm{x}, 1, i}^{\mathrm{e}} \mathcal{A}_{\mathrm{x}, 1, j}^{\mathrm{e}}}{s_{\mathrm{x}} p_{i \mid \mathrm{x}} p_{j \mid \mathrm{x}}}\right\}_{i, j=1}^{k_{\mathrm{x}}}
$$

Last but not least, I need to define $w^{(m)}$ and $n^{(m)}$. Recall that by following the analysis in Ref. [10], there is a one-one correspondence between a random variable in $\mathcal{W}$ taking the value of $\left\langle Q_{\mathrm{x}, i^{\prime}, j^{\prime}}\right\rangle_{i^{\prime}, j^{\prime}} \mathcal{A}_{\mathrm{x}, 1, i}^{\mathrm{e}} \mathcal{A}_{\mathrm{x}, 1, j}^{\mathrm{e}} /\left(s_{\mathrm{x}} p_{i \mid \mathrm{x}} p_{j \mid \mathrm{x}}\right)$ and an event that a photon pulse pair is prepared by Alice (Bob) using intensity $\mu_{\mathrm{x}, i}\left(\mu_{\mathrm{x}, j}\right)$ both in basis $\mathrm{X}$ and that the Bell basis measurement result announced by Charlie is inconsistent with the photon states prepared by Alice and Bob. Now let us arrange the $k_{\mathrm{x}}^{2}$ elements in the set $\mathcal{W}$ are arranged in descending order as $\left\{w^{(1)}, w^{(2)}, \cdots, w^{\left(k_{\mathrm{x}}^{2}\right)}\right\}$. Then, $n^{(i)}$ is the number of Bell basis measurement events that corresponds to the value of $w^{(i)} \in \mathcal{W}$.

There is an important subtlety that requires attention. In almost all cases of interest, each summard in Eq. (22) consists of three terms. The first two are positive and the third one is negative. The sum of the first two terms almost exactly equal to the magnitude of the third term. 
Hence, truncation error is very serious if one directly use Eq. (22) to numerically compute $\hat{r}$. The solution is to expand each term in powers of $1 / D_{m}$ and/or $1 / E_{m}$ defined below. This gives

$$
\approx \frac{y^{2} \operatorname{Width}(\mathcal{W})^{2}}{3} \sum_{m=1}^{k_{\mathrm{x}}^{2}} \frac{n^{(m)}}{D_{m} E_{m}}\left(\frac{1}{D_{m}^{2}}+\frac{1}{D_{m} E_{m}}+\frac{1}{E_{m}^{2}}\right),
$$

where

$$
D_{m}=y+\left(t-\sum_{i<m} n^{(i)}+1\right) x+\min \mathcal{W}+\sum_{i<m} n^{(i)} w^{(i)}
$$

and

$$
\begin{aligned}
E_{m}= & y+\left(t-\sum_{i<m} n^{(i)}+1\right) x+\min \mathcal{W}+\sum_{i<m} n^{(i)} w^{(i)} \\
& +n^{(m)}\left(w^{(m)}-x\right) \\
= & y+\left(t-\sum_{i \leq m} n^{(i)}+1\right) x+\min \mathcal{W}+\sum_{i \leq m} n^{(i)} w^{(i)} .
\end{aligned}
$$

(Note that only the leading term is kept in Eq. (24). This is acceptable because the next order term is of order of about 1/100 that of the leading term in all cases of practical interest.)

With all the above discussions, to summarize, the secure key rate $R$ of this $\epsilon_{\text {cor }}$-correct and $\epsilon_{\mathrm{sec}}$-secure QKD scheme in the finite raw key length situation is lowerbounded by

$$
\begin{aligned}
R \geq & \sum_{i, j=1}^{k_{\mathrm{z}}} \mathcal{B}_{\mathrm{Z}, i, j} Q_{\mathrm{Z}, i, j}-\left\langle Q_{\mathrm{Z}, i, j}\right\rangle_{i, j}\left[\frac{\ln \left(\chi / \epsilon_{\mathrm{sec}}\right)}{2 s_{\mathrm{Z}}}\right]^{1 / 2} \operatorname{Width}\left(\left\{\frac{\mathcal{B}_{\mathrm{Z}, i, j}}{p_{i \mid \mathrm{Z}} p_{j \mid \mathrm{Z}}}\right\}_{i, j=1}^{k_{\mathrm{z}}}\right)-p_{\mathrm{Z}}^{2}\left\{\langle\mu \exp (-\mu)\rangle_{\mathrm{z}}^{2} C_{\mathrm{z}, 2}^{2}\left[1-H_{2}\left(e_{p}\right)\right]\right. \\
& \left.+f_{\mathrm{EC}}\left\langle Q_{\mathrm{Z}, i, j} H_{s}\left(E_{\mathrm{Z}, i, j}\right)\right\rangle_{i, j}+\frac{\left\langle Q_{\mathrm{Z}, i, j}\right\rangle_{i, j}}{\ell_{\mathrm{raw}}}\left[6 \log _{2}\left(\frac{\chi}{\epsilon_{\mathrm{sec}}}\right)+\log _{2}\left(\frac{2}{\epsilon_{\mathrm{cor}}}\right)\right]\right\}
\end{aligned}
$$

and

$$
\begin{aligned}
& R \geq \sum_{i, j=1}^{k_{\mathrm{z}}} \mathcal{B}_{\mathrm{z}, i, j}^{\prime} Q_{\mathrm{z}, i, j}+\sum_{i, j=1}^{k_{\mathrm{x}}} \mathcal{B}_{\mathrm{x}, i, j} Q_{\mathrm{x}, i, j}-\left\langle Q_{\mathrm{z}, i, j}\right\rangle_{i, j}\left[\frac{\ln \left(\chi / \epsilon_{\mathrm{sec}}\right)}{2 s_{\mathrm{Z}}}\right]^{1 / 2} \operatorname{Width}\left(\left\{\frac{\mathcal{B}_{\mathrm{z}, i, j}^{\prime}}{p_{i \mid \mathrm{Z}} p_{j \mid \mathrm{Z}}}\right\}_{i, j=1}^{k_{\mathrm{z}}}\right)-\left\langle Q_{\mathrm{x}, i, j}\right\rangle_{i, j} \times \\
&\left.\quad \frac{\ln \left(\chi / \epsilon_{\mathrm{sec}}\right)}{2 s_{\mathrm{X}}}\right]^{1 / 2} \operatorname{Width}\left(\left\{\frac{\mathcal{B}_{\mathrm{x}, i, j}}{p_{i \mid \mathrm{x}} p_{j \mid \mathrm{X}}}\right\}_{i, j=1}^{k_{\mathrm{x}}}\right)-p_{\mathrm{Z}}^{2}\left\{\langle\mu \exp (-\mu)\rangle_{\mathrm{Z}}^{2} C_{\mathrm{x}, 2}^{2}\left[1-H_{2}\left(e_{p}\right)\right]+f_{\mathrm{EC}}\left\langle Q_{\mathrm{z}, i, j} H_{s}\left(E_{\mathrm{z}, i, j}\right)\right\rangle_{i, j}\right. \\
&\left.+\frac{\left\langle Q_{\mathrm{z}, i, j}\right\rangle_{i, j}}{\ell_{\mathrm{raw}}}\left[6 \log _{2}\left(\frac{\chi}{\epsilon_{\mathrm{sec}}}\right)+\log _{2}\left(\frac{2}{\epsilon_{\mathrm{cor}}}\right)\right]\right\}
\end{aligned}
$$

I remark that the R.H.S. of the above inequalities implicitly depends on $e_{\mathrm{X}, 1,1}$ whose upper bound obeys Inequalities (16), (17), (19), (21) and (24). Furthermore, when using the key rate in Inequality (26a), $\chi=9=$ $4+1+4$ for the first three inequalities concerning $e_{\mathrm{x} .1,1}$ and $\chi=10$ for the last inequality concerning $e_{\mathrm{X}, 1,1}[10]$. While using the key rate in Inequality (26b) instead of Inequality (26a), $\chi=9,10,10,11$ for Methods A, B, C and $\mathrm{D}$, respectively. (For reason for $\chi$ to increase by 1 except for Method A by switching the rate formula from Inequality (26a) to Inequality (26b) is due to the inclusion of the finite-size statistical fluctuations of the lower bound on $Y_{\mathrm{x}, 1,1}$.)

Compare with the corresponding key rate formula for standard QKD scheme, the most noticeable difference is the presence of additional terms and factors involving $C_{\mathrm{B}, 2}^{2}$ which tend to lower the key rate. Fortunately, $C_{\mathrm{B}, 2}$ roughly scale as $\mu_{\mathrm{B}, 1}^{k_{\mathrm{B}}}$ so that in practice, these terms and factors are negligible if $k_{\mathrm{B}} \gtrsim 2$ to 3 . Finally, I remark that the in the limit of $s_{Z} \rightarrow+\infty$, the key rate formulae in Inequalities (26a) and (26b) are tight in the sense that these lower bound are reachable although the condition for attaining them is highly unlikely to occur in realistic channels.

\section{PERFORMANCE ANALYSIS}

To study the key rate, I use the channel model reported by Ma and Razavi in Ref. 24], which I called the MR channel. For this channel,

$$
\begin{gathered}
Q_{\mathrm{X}, i, j}=2 \beta_{i j}^{2}\left[1+2 \beta_{i j}^{2}-4 \beta_{i j} I_{0}\left(\alpha_{\mathrm{x} i j}\right)+I_{0}\left(2 \alpha_{\mathrm{x} i j}\right)\right], \\
Q_{\mathrm{X}, i, j} E_{\mathrm{X}, i, j}=e_{0} Q_{\mathrm{x}, i, j}-2\left(e_{0}-e_{d}\right) \beta_{i j}^{2}\left[I_{0}\left(2 \alpha_{\mathrm{x} i j}\right)-1\right],
\end{gathered}
$$




$$
Q_{\mathrm{z}, i, j}=Q_{i j}^{(E)}+Q_{i j}^{(C)}
$$

and

$$
Q_{\mathrm{z}, i, j} E_{\mathrm{z}, i, j}=e_{d} Q_{i j}^{(C)}+\left(1-e_{d}\right) Q_{i j}^{(E)},
$$

where $I_{0}(\cdot)$ is the modified Bessel function of the first kind,

$$
\begin{gathered}
\alpha_{\mathrm{B} i j}=\frac{\sqrt{\eta_{\mathrm{A}} \mu_{\mathrm{B}, i} \eta_{\mathrm{B}} \mu_{\mathrm{B}, i}}}{2}, \\
\beta_{i j}=\left(1-p_{d}\right) \exp \left(-\frac{\eta_{\mathrm{A}} \mu_{\mathrm{x}, i}+\eta_{\mathrm{B}} \mu_{\mathrm{x}, j}}{4}\right), \\
e_{0}=\frac{1}{2}, \\
Q_{i j}^{(C)}=2\left(1-p_{d}\right)^{2} \exp \left(-\frac{\eta_{\mathrm{A}} \mu_{\mathrm{Z}, i}+\eta_{\mathrm{B}} \mu_{\mathrm{Z}, j}}{2}\right) \times \\
{\left[1-\left(1-p_{d}\right) \exp \left(-\frac{\eta_{\mathrm{A}} \mu_{\mathrm{Z}, i}}{2}\right)\right] \times} \\
{\left[1-\left(1-p_{d}\right) \exp \left(-\frac{\eta_{\mathrm{B}} \mu_{\mathrm{Z}, j}}{2}\right)\right]}
\end{gathered}
$$

and

$$
\begin{aligned}
Q_{i j}^{(E)}=2 p_{d}\left(1-p_{d}\right)^{2} \exp \left(-\frac{\eta_{\mathrm{A}} \mu_{\mathrm{Z}, i}+\eta_{\mathrm{B}} \mu_{\mathrm{Z}, j}}{2}\right) \times & \\
& {\left[I_{0}\left(2 \alpha_{\mathrm{Z} i j}\right)-\left(1-p_{d}\right) \exp \left(-\frac{\eta_{\mathrm{A}} \mu_{\mathrm{Z}, i}+\eta_{\mathrm{B}} \mu_{\mathrm{Z}, j}}{2}\right)\right] . }
\end{aligned}
$$

Here $e_{d}$ is the misalignment probability, $p_{d}$ is the dark count rate per detector. Moreover, $\eta_{\mathrm{A}}\left(\eta_{\mathrm{B}}\right)$ is transmittance of the channel between Alice (Bob) and Charlie. They are given by

$$
\eta_{\mathrm{A}}=\eta_{d} 10^{-\eta_{\mathrm{att}} L_{\mathrm{A}} / 10}
$$

and similarly for $\eta_{\mathrm{B}}$, where $L_{\mathrm{A}}$ is the length of the fiber connecting Alice to Charlie, $\eta_{d}$ is the detection efficiency of a detector, and $\eta_{\text {att }}$ is the transmission fiber loss constant.

I remark that the MR channel model assumes that the partial Bell state measurement is performed using linear optics with idea beam and/or polarization beam splitters. It also assumes that all photon detectors are identical and that the dead time is ignored. Moreover, this channel does not consider the use of quantum repeater.

The state-of-the-art key rate formula for decoy-state MDI-QKD with finite raw key length is the one by Mao et al. in Ref. [20], which extended an earlier result by Zhou et al. in Ref. [18]. (Note that even higher key rates have been reported by $\mathrm{Xu}$ et al. [16] and Zhou et al. [18]. Note however that the first work applied brute force optimization as well as the Chernoff bound on a much longer raw key. Its effectiveness in handling short

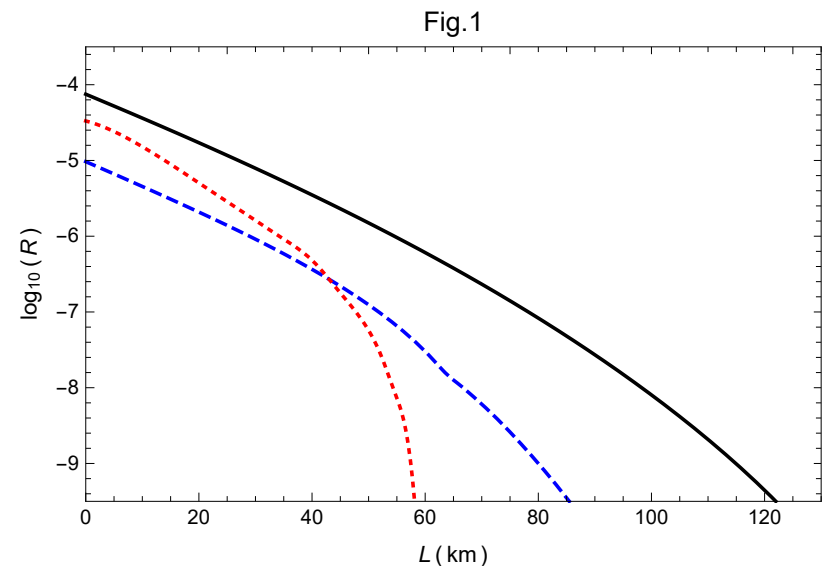

FIG. 1. Provably secure optimized key rates $R$ as a function of distance $L$ between Alice and Bob for $N_{t}=10^{10}$ and $\epsilon_{\mathrm{sec}} / \chi=$ $\epsilon_{\text {cor }}=10^{-10}$. The red dotted curve is the state-of-the-art provably secure key rate reported in Ref. [20]. The black solid curve is the rate computed for $(3,2)_{G}$; and the blue dashed curve is rate for $(3,3)_{R}$. The rates for $\left(k_{\mathrm{x}} \geq 3, k_{\mathrm{Z}} \geq 3\right)_{G}$ and $\left(k_{\mathrm{x}} \geq 4, k_{\mathrm{z}} \geq 4\right)_{R}$ is higher than that of $(3,2)_{G}$ by about $33 \%$. But they are omitted here as those curves would visually almost overlap with the black solid curve in this semi-log plot.

raw key length situation is not apparent. Whereas the second work assumed that the statistical fluctuation is Gaussian distributed which is not justified in terms of unconditional security.) To compare with the provably secure key rate reported in Ref. [20], I use their settings by putting $e_{d}=1.5 \%, p_{d}=6.02 \times 10^{-6}, \eta_{\text {att }}=0.2 \mathrm{db} / \mathrm{km}$, $\eta_{d}=14.5 \%, f_{\mathrm{EC}}=1.16$, and $L_{\mathrm{A}}=L_{\mathrm{B}}=L / 2$ where $L$ is the transmission distance between Alice and Bob. For the security parameters, I follow Ref. [20] by setting $\epsilon_{\mathrm{sec}} / \chi=10^{-10}$ although a more meaningful way is to set $\epsilon_{\mathrm{sec}}$ divided by the length of the final key to a fixed small number 11. Whereas for $\epsilon_{\mathrm{cor}}$, its value has not been specified in Refs. 20]. Fortunately, Inequality (26a) implies that the provably secure key rate does not sensitively dependent on $\epsilon_{\text {cor }}$. Here I simply set it to $10^{-10}$.

Fig. 1 compares the key rates when the total number of photon pulse pairs prepared by Alice and Bob, $N_{t} \approx \ell_{\text {raw }} /\left(p_{\mathrm{Z}}^{2}\left\langle Q_{\mathrm{z}, i, j}\right\rangle_{i, j}\right)$ is set to $10^{10}$. For each of the curves, the number of photon intensities $k_{\mathrm{X}}\left(k_{\mathrm{z}}\right)$ used for $\mathrm{X}(\mathrm{Z})$ are fixed. The smallest photon intensities $\mu_{\mathrm{X}, k_{\mathrm{X}}}$ and $\mu_{\mathrm{z}, k_{\mathrm{z}}}$ are both set to be $10^{-6}$. The optimized key rate is then calculated by varying the other $\mu_{\mathrm{x}, i}$ 's, $\mu_{\mathrm{z}, i}$ 's as well as $p_{i \mid \mathrm{X}}, p_{i \mid \mathrm{Z}}$ and $p_{Z}$ by a combination of random sampling (with a minimum of $10^{7}$ samples to a maximum of about $10^{9}$ samples per data point on each curve) and adaptive gradient descend method (that is, the step size is adjusted dynamically to speed up the descend). For some of the curves, I introduce additional constraints that $\mu_{\mathrm{x}, i}=\mu_{\mathrm{z}, i}$ so as to reduce the number of different photon intensities used. To aid discussion, I refer to the unconstrained and constrained situations by $\left(k_{\mathrm{x}}, k_{\mathrm{z}}\right)_{G}$ and $\left(k_{\mathrm{x}}, k_{\mathrm{z}}\right)_{R}$, respectively. 
Fig.2

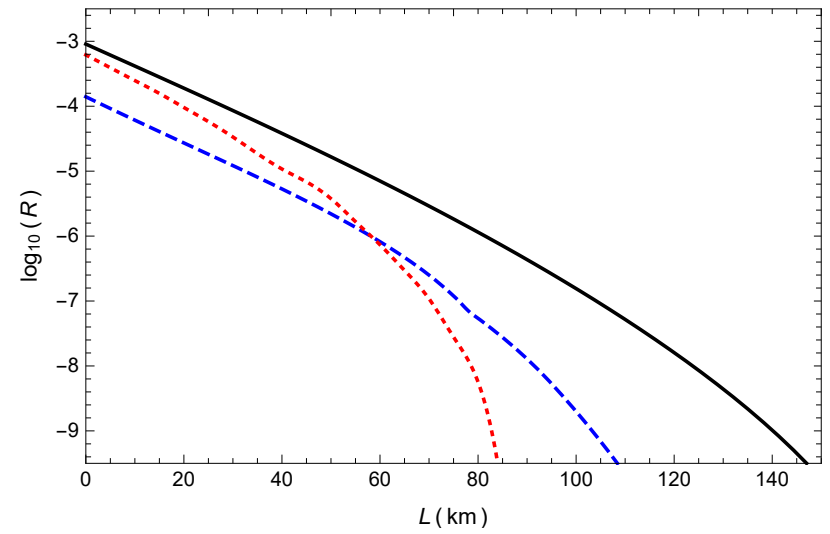

FIG. 2. Provably secure optimized key rates $R$ as a function of distance $L$ between Alice and Bob for $N_{t}=10^{9}, \epsilon_{\mathrm{sec}} / \chi=$ $\epsilon_{\text {cor }}=10^{-7}$. The red dotted curve is the provably secure key rate reported in Ref. [18]. The black solid curve is the rate computed for $(3,2)_{G}$; and the blue dashed curve is rate for $(3,3)_{R}$. The rates for $\left(k_{\mathrm{X}} \geq 3, k_{\mathrm{Z}} \geq 3\right)_{G}$ are about the same as that of $(3,2)_{G}$ while those of $\left(k_{\mathrm{X}} \geq 4, k_{\mathrm{Z}} \geq 4\right)_{R}$ are a little bit lower (higher) than that of $(3,2)_{G}$ for short (long) transmission distances. They are omitted here to avoid curve jamming.

The $R-L$ graphs in Fig. 1 clearly show the advantage of using the method in this text in computing the provably secure (optimized) key rate. The black curve, which is the distance-rate graph of $(3,2)_{G}$ that uses four different photon intensities, is much better than the red one (which also uses four different photon intensities) originally reported in Ref. [20]. In fact, for any distance $L$ between Alice and Bob, the key rate of the $(3,2)_{G}$ method is at least 2.25 times that of the state-of-the-art key rate reported in Ref. [20]. (I also mention on passing that the rate of the black curve is even higher than that of the two decoy key rate reported in Ref. [16] using a much longer raw key length of $\ell_{\text {raw }}=10^{12}$.) Besides, the $(3,2)_{G}$ method extends the working distance between Alice and Bob from slightly less than $60 \mathrm{~km}$ to slightly over $130 \mathrm{~km}$. The blue dashed curve is the key rate of $(3,3)_{R}$, which uses the same set of three different photon intensities for both preparation bases. Although it uses one less photon intensity, it still outperforms the key rate of the red curve when $L \gtrsim 45 \mathrm{~km}$.

To further illustrate the power of this method, I compare the key rates here with the ones obtained in Ref. [18] in which they used four photon states and the following paramters: $e_{d}=1.5 \%, p_{d}=10^{-7}, \eta_{\text {att }}=0.2 \mathrm{db} / \mathrm{km}$, $\eta_{d}=40 \%, f_{\mathrm{EC}}=1.16, L_{\mathrm{A}}=L_{\mathrm{B}}=L / 2$ and $\epsilon_{\mathrm{sec}} / \chi=$ $\epsilon_{\text {cor }}=10^{-7}$. The optimized key rates are then found using the same method that produces Fig. 1. As shown in Fig. 2, the optimized key rate of $(3,2)_{G}$ (the black curve that uses four different photon intensities) is at least $90 \%$ higher than those reported in Ref. [18]. Just like the previous comparison, the key rate of $(3,3)_{R}$ which uses only three different photon intensities is better than the one

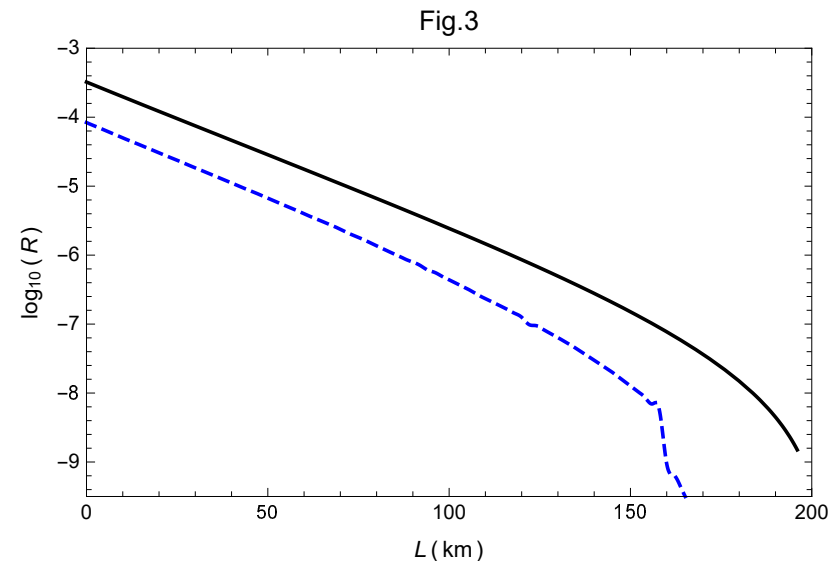

FIG. 3. Provably secure optimized key rates $R$ as a function of distance $L$ between Alice and Bob for $\ell_{\text {raw }}=10^{10}$ and $\kappa=10^{-15}$ and $\epsilon_{\text {cor }}=10^{-10}$. The black solid curve is for $(3,2)_{G}$ and the blue dashed curve is for $(3,3)_{R}$. The rates for $\left(k_{\mathrm{x}} \geq 3, k_{\mathrm{z}} \geq 3\right)_{G}$ and $\left(k_{\mathrm{x}} \geq 4, k_{\mathrm{z}} \geq 4\right)_{R}$ is higher than that of $(3,2)_{G}$ by about $18 \%$. These additional curves are omitted here as visually they almost overlap with the black solid curve in this semi-log plot.

reported in Ref. 18] when $L \gtrsim 60 \mathrm{~km}$. Last but not least, the maximum transmission distance increases from about $87 \mathrm{~km}$ to about $156 \mathrm{~km}$ for $(3,2)_{G}$ and $162 \mathrm{~km}$ for $(4,3)_{G}$ (the later not sure in the figure to avoid curve crowding).

In a sense, instead of $\epsilon_{\mathrm{sec}} / \chi$, a fairer security parameter to use is $\kappa$, namely $\epsilon_{\text {sec }}$ per number of bits of the final key [1]. Fig. 3 depicts the $R-L$ curves of various methods when $\kappa=10^{-15}$ and $\epsilon_{\text {cor }}=10^{-10}$. Here instead of fixing $N_{t}$, I keep $\ell_{\text {raw }}=10^{10}$ which corresponds to a much greater value of $N_{t}$ in general. The blue dashed curve is the rate for $(3,3)_{R}$ which uses three photon intensities. It already achieves a non-zero key rate at a distance of slightly greater than $160 \mathrm{~km}$. The black curve is the rate for $(3,2)_{G}$ which uses four photon intensities. It allows Alice and Bob to share a secret key over a distance close to $200 \mathrm{~km}$. This finding makes sense for a larger raw key length $\ell_{\text {raw }}$ implies smaller statistical fluctuations in our estimates of various yields and error rate, which in turn increase the provably secure key rate and the maximum transmission distance.

Tables [1] and II] shows the provably secure optimized key rates using various values of $k_{\mathrm{X}}$ and $k_{\mathrm{Z}}$ for the case of fixing $\epsilon_{\mathrm{sec}} / \chi$ and $\kappa$, respectively. The following points can be drawn from these figures and tables. First, for the unconstrained photon intensity situation, the optimized key rate increases as $k_{\mathrm{X}}$ increases. For instance, as shown in Table I, the key rate of $(4,2)_{G}$ is at least $39 \%$ higher than that of $(3,2)_{G}$ by fixing $\epsilon_{\mathrm{sec}} / \chi$. And from Table II the corresponding increase in key rate by fixing $\kappa$ is about $18 \%$ in the distance range from $0 \mathrm{~km}$ to $150 \mathrm{~km}$. (I do not draw these curves in Figs. 1 and 3 because they almost overlap with the $(3,2)_{G}$ curve using the same plotting scales.) Second, for the constrained photon intensity 


\begin{tabular}{||c|c|c||}
\hline \hline$L / \mathrm{km}$ & 0 & 50 \\
\hline$(3,2)_{G}$ & $7.49 \times 10^{-5}$ & $1.50 \times 10^{-6}$ \\
\hline$(3,3)_{R}$ & $9.65 \times 10^{-6}$ & $1.25 \times 10^{-7}$ \\
\hline$(3,3)_{G}$ & $8.51 \times 10^{-5}$ & $1.82 \times 10^{-6}$ \\
\hline$(4,2)_{G}$ & $1.04 \times 10^{-4}$ & $2.22 \times 10^{-6}$ \\
\hline$(4,3)_{G}$ & $1.04 \times 10^{-4}$ & $2.24 \times 10^{-6}$ \\
\hline$(4,4)_{R}$ & $3.10 \times 10^{-5}$ & $3.75 \times 10^{-7}$ \\
\hline$(4,4)_{G}$ & $1.04 \times 10^{-4}$ & $2.23 \times 10^{-6}$ \\
\hline \hline
\end{tabular}

TABLE I. Optimized secure key rates for $N_{t}=10^{10}$ and $\epsilon_{\mathrm{sec}} / \chi=\epsilon_{\mathrm{cor}}=10^{-10}$.

situation, the optimized key rate increases as $k_{\mathrm{x}}=k_{\mathrm{z}}$ increases. These findings can be understood by the fact that the more decoy intensity used, the closer the various bounds of yields and error rates are to their actual values. Third, the constrained key rate is in general several times lower than the corresponding unconstrained one. So, using the same set of photon intensities for the two bases is not a good idea, at least for the MR channel.

There is an interesting observation that requires indepth explanation. From Table II for the case of fixing $k_{\mathrm{X}}$ and $\kappa$, the increase in $R$ due to increase in $k_{\mathrm{Z}}$ is insignificant. Moreover, Table [1 shows that for the case fixing $\epsilon_{\mathrm{sec}} / \chi$ and $k_{\mathrm{X}}$, significant increase in key rate occurs only when $k_{\mathrm{x}}=3$. The reason is that for the MR channel 24], it turns out that the key rate computed by Inequality (26b) is greater than that computed by Inequality (26a). That is to say, the lower bound of $Y_{\mathrm{X}, 1,1}$ is a better estimate of the single photon pair yield that the lower bound of $Y_{\mathrm{z}, 1,1}$. Thus, increasing $k_{\mathrm{z}}$ only gives a better estimate of $Y_{z, 0, \star}$. Since I fix the lowest photon intensity to $10^{-6}$, which is very close to the vacuum state, the major error in estimating $Y_{\mathrm{z}, 0, \star}$ comes from finite-size statistical fluctuation. Consequently, by fixing a large enough raw key length $\ell_{\text {raw }}$, the use of more than two photon intensities for the $\mathbf{Z}$ does not improve the provably secure key rate in practice. In other words, the improvement on the provably secure key rate by increasing $k_{\mathrm{Z}}$ alone for the MR channel occurs only when $k_{\mathrm{Z}}$ is small, say, about 2 to 3 and when $\ell_{\text {raw }}$ is small.

There is a systematic trend that worth reporting. For the case of using unconstrained photon intensities, Method D plus the use of $Y_{\mathrm{X}, 1,1}$ to bound the single photon-pair yield gives the highest key rate over almost the whole range of distance $L$. Only when close to the maximum transmission distance that the best rate is computed using Method $\mathrm{C}$ and $Y_{\mathrm{x}, 1,1}$. Whereas for the case of constrained photon intensities, for short transmission distance, the best rate is computed using Method D plus $Y_{\mathrm{Z}, 1,1}$. For longer transmission distance, the best rate is due to Method $\mathrm{B}$ and $Y_{\mathrm{x}, 1,1}$.

\begin{tabular}{||c|c|c|c|c||}
\hline \hline$L / \mathrm{km}$ & 0 & 50 & 100 & 150 \\
\hline$(3,2)_{G}$ & $3.23 \times 10^{-4}$ & $2.85 \times 10^{-5}$ & $2.44 \times 10^{-6}$ & $1.51 \times 10^{-7}$ \\
\hline$(3,3)_{R}$ & $8.37 \times 10^{-5}$ & $6.67 \times 10^{-6}$ & $4.33 \times 10^{-7}$ & $1.27 \times 10^{-8}$ \\
\hline$(3,3)_{G}$ & $3.23 \times 10^{-4}$ & $2.85 \times 10^{-5}$ & $2.44 \times 10^{-6}$ & $1.51 \times 10^{-7}$ \\
\hline$(4,2)_{G}$ & $3.82 \times 10^{-4}$ & $3.39 \times 10^{-5}$ & $2.89 \times 10^{-6}$ & $1.78 \times 10^{-7}$ \\
\hline$(4,3)_{G}$ & $3.82 \times 10^{-4}$ & $3.39 \times 10^{-5}$ & $2.89 \times 10^{-6}$ & $1.78 \times 10^{-7}$ \\
\hline$(4,4)_{R}$ & $1.70 \times 10^{-4}$ & $1.32 \times 10^{-5}$ & $8.27 \times 10^{-7}$ & $2.64 \times 10^{-8}$ \\
\hline$(4,4)_{G}$ & $3.82 \times 10^{-4}$ & $3.39 \times 10^{-5}$ & $2.89 \times 10^{-6}$ & $1.78 \times 10^{-7}$ \\
\hline \hline
\end{tabular}

TABLE II. Optimized secure key rates for $\ell_{\text {raw }}=10^{10}, \kappa=$ $10^{-15}$ and $\epsilon_{\mathrm{cor}}=10^{-10}$.

\section{SUMMARY}

In summary, using the BB84 scheme in the MR channel as an example, I have reported a key rate formula for MDI-QKD using Possionian photon sources through repeated use the inversion of Vandermonde matrix and a McDiarmid-type inequality. This method gives a provably secure key rate that is at least 2.25 times that of the current state-of-the-art result. It also shows that using five photon intensities, more precisely the $(4,2)$-method, gives an additional $18 \%$ increase in the key rate for the MR channel. This demonstrates once again the effectiveness of using McDiarmid-type inequality in statistical data analysis in physical problems. Provided that the photon source is sufficiently close to Possionian,

Note that the Vandermonde matrix inversion technique is rather general. As pointed out in Remark 1 in the Appendix, by modifying the proof of Lemma 2 one can show that $C_{3 i} \geq 0$ if $k$ is even and $C_{3 i}<0$ if $k$ is odd for all $i \geq k$. Thus, I can find the lower bound of $Y_{\mathrm{B}, 0,2}$ and $Y_{\mathrm{B}, 2,0}$. In other words, I can extend the key rate calculation to the case of twin-field [25] or phase-matching MDI-QKD [26]. Note further that Inequalities (44) are still valid by replacing the $\mathcal{A}_{\mathrm{B}, j, i}^{\mathrm{e}}$ 's and $\mathcal{A}_{\mathrm{B}, j, i}^{\mathrm{o}}$ 's by their perturbed expressions through standard matrix inversion perturbation as long as the photon sources are sufficiently close to Possionian. In this regard, the theory developed here also applies to these sources. Interested readers are invited to fill in the details. Last but not least, it is instructive to extend this work to cover other MDI-QKD protocols as well as more realistic quantum channels that take dead time and imperfect beam splitters into account.

\section{Appendix A: Auxiliary Results On Bounds Of Yields And Error Rates}

I begin with the following lemma.

Lemma 1. Let $\mu_{1}, \mu_{2}, \cdots, \mu_{k}$ be $k \geq 2$ distinct real numbers. Then

$$
\sum_{i=1}^{k} \frac{\mu_{i}^{\ell}}{\prod_{t \neq i}\left(\mu_{i}-\mu_{t}\right)}=0
$$


for $\ell=0,1, \cdots, k-2$.

Proof. Note that the L.H.S. of Eq. A1 is a symmetric function of $\mu_{i}$ 's. Moreover, only its first two terms involve the factor $\mu_{1}-\mu_{2}$ in the denominator. In fact, the sum of the these two terms equals

$$
\frac{\mu_{1}^{\ell} \prod_{t>2}\left(\mu_{2}-\mu_{t}\right)-\mu_{2}^{\ell} \prod_{t>2}\left(\mu_{1}-\mu_{t}\right)}{\left(\mu_{1}-\mu_{2}\right) \prod_{t>2}\left[\left(\mu_{1}-\mu_{t}\right)\left(\mu_{2}-\mu_{t}\right)\right]} .
$$

By applying reminder theorem, I know that the numerator of the above expression is divisible by $\mu_{1}-\mu_{2}$. Consequently, the L.H.S. of Eq. (A1) is a homogeneous polynomial of degree $\leq \ell-k+1$. But as $\ell \leq k-2$, this means the L.H.S. of Eq. (A1) must be a constant. By putting $\mu_{i}=t^{i}$ for all $i$ and by taking the limit $t \rightarrow+\infty$, I conclude that this constant is 0 . This completes the proof.

Following the notation in Ref. [9], I define

$$
C_{a+1, i}=\frac{(-1)^{k-a} a !}{i !} \sum_{t=1}^{k} \frac{\mu_{t}^{i} S_{t a}}{\prod_{\ell \neq t}\left(\mu_{t}-\mu_{\ell}\right)}
$$

where

$$
S_{t a}=\sum^{\prime} \mu_{t_{1}} \mu_{t_{2}} \cdots \mu_{t_{k-a-1}}
$$

with the primed sum being over all $t_{j}$ 's $\neq i$ obeying $1 \leq$ $t_{1}<t_{2}<\cdots<t_{k-a-1} \leq k$. The following lemma is an extension of a result in Ref. [9].

Lemma 2. Let $\mu_{1}>\mu_{2}>\cdots>\mu_{k} \geq 0$. Suppose $0 \leq i<k$. Then

$$
C_{a+1, i}= \begin{cases}-1 & \text { if } a=i \\ 0 & \text { otherwise }\end{cases}
$$

Whereas if $i \geq k$, then

$$
\begin{cases}C_{1 i} \geq 0 \text { and } C_{2 i}<0 & \text { if } k \text { is even } \\ C_{1 i} \leq 0 \text { and } C_{2 i}>0 & \text { if } k \text { is odd }\end{cases}
$$

Proof. Using the same argument as in the proof of Lemma 1, I conclude that $C_{a+1, i}$ is a homogeneous polynomial of degree $\leq i-a$.

Consider the case of $i \leq a$ so that $C_{a+1, i}$ is a constant. By putting $\mu_{t}=\delta^{t}$ for all $t$ and then taking the limit $\delta \rightarrow 0^{+}$, it is straightforward to check that $C_{a+1, i}=0$ if $i<a$ and $C_{a+1, i}=-1$ if $i=a$.

It remains to consider the case of $a<i<k$. I first consider the subcase of $a=0$. Here $C_{1 i}$ contains a common factor of $\prod_{t=1}^{k} \mu_{t}$, which is of degree $k>i$. Therefore, I could write $\prod_{t=1}^{k} \mu_{t}=C_{1 i} F$ where $F$ is a homogeneous polynomial of degree $\geq i$. As a consequence, either $C_{1 i}$ or $F$ contains $\mu_{1}$ and hence all $\mu_{t}$ 's. Thus, $C_{1 i}$ must be a constant for $i<k$. By setting $\mu_{k}=0$, I know that $C_{1 i}=0$.
Next, I consider the subcase of $a=1$. Since $i>1$, from the findings of the first subcase, I arrive at

$$
\begin{aligned}
C_{2 i} & =\frac{(-1)^{k-1}}{i !} \sum_{t=1}^{k} \frac{T_{1} \mu_{t}^{i-1}-\left(\prod_{\ell=1}^{k} \mu_{\ell}\right) \mu_{t}^{i-2}}{\prod_{\ell \neq t}\left(\mu_{t}-\mu_{\ell}\right)} \\
& =\frac{(-1)^{k-1} T_{1}}{i !} \sum_{t=1}^{k} \frac{\mu_{t}^{i-1}}{\prod_{\ell \neq t}\left(\mu_{t}-\mu_{\ell}\right)}-C_{1, i-1} \\
& =\frac{(-1)^{k-1} T_{1}}{i !} \sum_{t=1}^{k} \frac{\mu_{t}^{i-1}}{\prod_{\ell \neq t}\left(\mu_{t}-\mu_{\ell}\right)},
\end{aligned}
$$

where $T_{1}$ is the symmetric polynomial

$$
T_{1}=\sum_{t=1}^{k} \mu_{1} \mu_{2} \cdots \mu_{t-1} \mu_{t+1} \cdots \mu_{k}
$$

By Lemma 1, I find that $C_{2 i}=0$ as $i<k$.

The third subcase I consider is $a=2$. As $i>2$,

$$
C_{3 i}=\frac{2(-1)^{k}}{i !} \sum_{t=1}^{k} \frac{T_{2} \mu_{t}^{i-1}-T_{1} \mu_{t}^{i-2}+\left(\prod_{\ell=1}^{k} \mu_{\ell}\right) \mu_{t}^{i-3}}{\prod_{\ell \neq t}\left(\mu_{t}-\mu_{\ell}\right)}
$$

where

$$
T_{2}=\sum^{\prime} \mu_{t_{1}} \mu_{t_{2}} \cdots \mu_{t_{k-2}}
$$

with the primed sum over all $t_{j}$ 's with $1 \leq t_{1}<t_{2}<$ $\cdots<t_{k-2} \leq k$. By Lemma 1] I get $C_{3 i}=0$ as $i<k$.

By induction, the proof of the subcase $a=2$ can be extended to show the validity for all $a \leq k$ and $a<i<k$. This shows the validity of Eq. (A4)

The proof of Eq. A5 can be found in Ref. [9]. I reproduce here for easy reference. By expanding the $1 /\left(\mu_{1}-\mu_{t}\right)$ 's in $C_{a i}$ as a power series of $\mu_{1}$ with all the other $\mu_{t}$ 's fixed, I obtain

$$
\begin{gathered}
C_{1 i}=\frac{(-1)^{k}}{i !}\left(\prod_{t=1}^{k} \mu_{t}\right)\left[\mu_{1}^{i-k} \prod_{r=2}^{k}\left(1+\frac{\mu_{r}}{\mu_{1}}+\frac{\mu_{r}^{2}}{\mu_{1}^{2}}+\cdots\right)\right. \\
\left.+f\left(\mu_{2}, \mu_{3}, \cdots, \mu_{k}\right)\right]+\mathrm{O}\left(\frac{1}{\mu_{1}}\right)
\end{gathered}
$$

for some function $f$ independent of $\mu_{1}$. As $C_{1 i}$ is a homogeneous polynomial of degree $\leq i$, by equating terms in powers of $\mu_{1}$, I get

$$
C_{1 i}=\frac{(-1)^{k}}{i !}\left(\prod_{t=1}^{k} \mu_{t}\right) \sum_{\substack{t_{1}+t_{2}+\cdots+t_{k}=i-k, t_{1}, t_{2}, \cdots, t_{k} \geq 0}} \mu_{1}^{t_{1}} \mu_{2}^{t_{2}} \cdots \mu_{k}^{t_{k}}
$$

for all $i \geq k$. As all $\mu_{t}$ 's are non-negative, $C_{1 i} \geq 0$ if $k$ is even and $C_{1 i} \leq 0$ if $k$ is odd. 
By the same argument, I expand all the $1 /\left(\mu_{1}-\mu_{t}\right)$ terms in $C_{2 i}$ in powers of $\mu_{1}$ to get

$$
\begin{aligned}
C_{2 i}= & \frac{(-1)^{k-1} T_{1}}{i !} \sum_{\substack{t_{1}+t_{2}+\cdots+t_{k}=i-k \\
t_{1}>0, t_{2}, \cdots, t_{k} \geq 0}} \mu_{1}^{t_{1}} \mu_{2}^{t_{2}} \cdots \mu_{k}^{t_{k}} \\
& +f^{\prime}\left(\mu_{2}, \cdots, \mu_{k}\right)
\end{aligned}
$$

for some function $f^{\prime}$ independent of $\mu_{1}$. By recursively expanding Eq. (A2) in powers of $\mu_{2}$ but with $\mu_{1}$ set to 0 , and then in powers of $\mu_{3}$ with $\mu_{1}, \mu_{2}$ set to 0 and so on, I conclude that whenever $i \geq k$, then $C_{2 i}<0$ if $k$ is even and $C_{2 i}>0$ if $k$ is odd. This completes the proof.

Remark 1. By the same technique of expanding each factor of $1 /\left(\mu_{1}-\mu_{t}\right)$ in $C_{a+1, i}$ in powers of $\mu_{1}$, it is straightforward to show that if $i \geq k$ and $j \geq 1$, then $C_{2 j+1, i} \geq 0$ and $C_{2 j, i} \leq 0$ provided that $k$ is even. And $C_{2 j+1, i} \leq 0$ and $C_{2 j, i} \geq 0$ provided that $k$ is odd.

The following theorem is an extension of a similar result reported in Ref. [9] by means of an explicit expression of the inverse of a Vandermonde matrix.

Theorem 1. Let $\mu_{1}>\mu_{2}>\cdots>\mu_{k} \geq 0$ and $\tilde{\mu}_{1}>$ $\tilde{\mu}_{2}>\cdots>\tilde{\mu}_{\tilde{k}} \geq 0$. Suppose

$$
\sum_{a, b=0}^{+\infty} \frac{\mu_{i}^{a}}{a !} \frac{\tilde{\mu}_{j}^{b}}{b !} A_{a b} \equiv \sum_{a, b=0}^{+\infty} M_{a+1, i} \tilde{M}_{b+1, j} A_{a b}=B_{i j}
$$

for all $i=1,2, \cdots, k$ and $j=1,2, \cdots, \tilde{k}$. Then,

$$
\begin{aligned}
A_{a b}= & \sum_{i=1}^{k} \sum_{j=1}^{\tilde{k}}\left(M^{-1}\right)_{a+1, i}\left(\tilde{M}^{-1}\right)_{b+1, j} B_{i j} \\
& +\sum_{I=k}^{+\infty} C_{a+1, I} A_{I b}+\sum_{J=\tilde{k}}^{+\infty} \tilde{C}_{b+1, J} A_{a J} \\
& -\sum_{I=k}^{+\infty} \sum_{J=\tilde{k}}^{+\infty} C_{a+1, I} \tilde{C}_{b+1, J} A_{I J}
\end{aligned}
$$

for all $a=0,1, \cdots, k-1$ and $b=0,1, \cdots, \tilde{k}-1$. Here

$$
\left(M^{-1}\right)_{a+1, i}=\frac{(-1)^{k-a-1} a ! S_{i a}}{\prod_{t \neq i}\left(\mu_{i}-\mu_{t}\right)}
$$

and similarly for $\left(\tilde{M}^{-1}\right)_{b+1, j}$.

Proof. Note that for any fixed $a=0,1, \cdots, k-1$ and $b=0,1, \cdots, \tilde{k}-1$,

$\sum_{b=0}^{+\infty} \frac{\tilde{\mu}_{j}^{b}}{b !} A_{a b}=\sum_{i=1}^{k}\left(M^{-1}\right)_{a+1, i}\left(B_{i j}-\sum_{b=0}^{+\infty} \sum_{I=k}^{+\infty} \frac{\mu_{i}^{I}}{I !} \frac{\tilde{\mu}_{j}^{b}}{b !} A_{I b}\right)$.

Here $M^{-1}$ is the inverse of the $k \times k$ matrix $\left(M_{a+1, i}\right)_{a+1, i=1}^{k}$. From Ref. [9], the matrix elements of
$M^{-1}$ are related to inverse of certain Vandermonde matrix and are given by the expression immediately after Eq. (A14). From Lemma 2, Eq. (A16) can be rewritten as

$$
\sum_{b=0}^{+\infty} \frac{\tilde{\mu}_{j}^{b}}{b !} A_{a b}=\sum_{a=1}^{k}\left(M^{-1}\right)_{a+1, i} B_{i j}+\sum_{b=0}^{+\infty} \sum_{I=k}^{+\infty} \frac{\tilde{\mu}_{j}^{b}}{b !} C_{a+1, I} A_{I b} .
$$

By repeating the above procedure again, I find that for any fixed $a=0,1, \cdots, k-1$ and $b=0,1, \cdots, \tilde{k}-1$,

$$
\begin{aligned}
A_{a b}= & \sum_{i=1}^{k} \sum_{j=1}^{\tilde{k}}\left(M^{-1}\right)_{a+1, i}\left(\tilde{M}^{-1}\right)_{b+1, j} B_{i j} \\
& -\sum_{I=k}^{+\infty} \sum_{\tilde{t}=0}^{+\infty} C_{a+1, I} \tilde{C}_{b+1, \tilde{t}} A_{I \tilde{t}}+\sum_{J=\tilde{k}}^{+\infty} \tilde{C}_{b+1, J} A_{a J} .
\end{aligned}
$$

Here the $\tilde{k} \times \tilde{k}$ matrix $\tilde{C}$ is defined in the exactly the same as the $k \times k$ matrix $C$ except that the $k$ and $\mu_{t}$ 's variables are replaced by $\tilde{k}$ and the corresponding $\tilde{\mu}_{t}$ 's. Substituting Eq. (A4) into the above equation gives Eq. (A14).

Applying Lemma 2 and Theorem 1, in particular, the Inequality (A5), I arrive at the following two Corollaries.

Corollary 1. Suppose the conditions stated in Theorem 1 are satisfied. Suppose further that $A_{a b}=0$ for all $b>0$ and $a \geq 0$; and $A_{a 0} \in[0,1]$ for all $a$. Then

$$
A_{00} \geq \sum_{i=1}^{k}\left(M^{-1}\right)_{1 i} B_{i 0}
$$

Corollary 2. Suppose the conditions stated in Theorem 1 are satisfied. Suppose further that $A_{a b} \in[0,1]$ for all $a, b$. Then

$$
A_{00} \geq \sum_{i=1}^{k} \sum_{j=1}^{\tilde{k}}\left(M^{-1}\right)_{1 i}\left(\tilde{M}^{-1}\right)_{1 j} B_{i j}-\sum_{I=k}^{+\infty} \sum_{J=\tilde{k}}^{+\infty} C_{1 I} \tilde{C}_{1 J}
$$

and

$$
A_{11} \leq \sum_{i=1}^{k} \sum_{j=1}^{\tilde{k}}\left(M^{-1}\right)_{2 i}\left(\tilde{M}^{-1}\right)_{2 j} B_{i j}
$$

provided both $k$ and $\tilde{k}$ are even. Furthermore,

$$
A_{11} \geq \sum_{i=1}^{k} \sum_{j=1}^{\tilde{k}}\left(M^{-1}\right)_{2 i}\left(\tilde{M}^{-1}\right)_{2 j} B_{i j}-\sum_{I=k}^{+\infty} \sum_{J=\tilde{k}}^{+\infty} C_{2 I} \tilde{C}_{2 J}
$$

if both $k$ and $\tilde{k}$ are odd.

Remark 2. Clearly, each of the bounds in the above Corollary are tight. Although the conditions for attaining the bound in Inequality (A20b) are not compatible 
with those for attaining the bounds in Inequalities A20a and (A20c), the way I use these inequalities in Secs. $I V$ and $\nabla$ ensures that it is possible to attaining all these bounds in the key rate formula.

\section{ACKNOWLEDGMENTS}

This work is supported by the RGC grant 17302019 of the Hong Kong SAR Government.
[1] X.-B. Wang, Phys. Rev. Lett. 94, 230503 (2005).

[2] H.-K. Lo, X. Ma, and K. Chen, Phys. Rev. Lett. 94, 230504 (2005).

[3] H.-K. Lo, M. Curty, and B. Qi, Phys. Rev. Lett. 108, 130503 (2012).

[4] U. Vazirani and T. Vidick, Phys. Rev. Lett. 113, 140501 (2014).

[5] K. Brádler, M. Mirhosseini, R. Fickler, A. Broadbent, and R. Boyd, New J. Phys. 18, 073030 (2016).

[6] L. Dellantonio, A. S. Sørensen, and B. Bacco, Phys. Rev. A 98, 062301 (2018).

[7] M. Hayashi, New J. Phys. 9, 284 (2007).

[8] M. Hayashi and R. Nakayama, New J. Phys. 16, 063009 (2014).

[9] H. F. Chau, Phys. Rev. A 97, 040301(R) (2018).

[10] H. F. Chau and K. C. J. Ng, New J. Phys. (2020), in press.

[11] C. C. W. Lim, M. Curty, N. Walenta, F. Xu, and H. Zbinden, Phys. Rev. A 89, 022307 (2014).

[12] X. Ma, C.-H. F. Fung, and M. Razavi, Phys. Rev. A 86, 052305 (2012).

[13] S.-H. Sun, M. Gao, C.-Y. Li, and L.-M. Liang, Phys. Rev. A 87, 052329 (2013).

[14] S.-H. Sun, M. Gao, C.-Y. Li, and L.-M. Liang, Phys. Rev. A 88, 019905 (2013).

[15] M. Curty, F. Xu, W. Cui, C. C. W. Lim, K. Tamaki, and H.-K. Lo, Nat. Comm. 5, 372 (2014).

[16] F. Xu, H. Xu, and H.-K. Lo, Phys. Rev. A 89, 052333 (2014).

[17] Z.-W. Yu, Y.-H. Zhou, and X.-B. Wang, Phys. Rev. A 91, 032318 (2015).

[18] Y.-H. Zhou, Z.-W. Yu, and X.-B. Wang, Phys. Rev. A
93, 042324 (2016).

[19] Z. Zhang, Q. Zhao, M. Razavi, and X. Ma, Phys. Rev. A 95, $012333(2017)$.

[20] C.-C. Mao, X.-Y. Zhou, J.-R. Zhu, C.-H. Zhang, C.-M. Zhang, and Q. Wang, Opt. Express 26, 13289 (2018).

[21] Y. Wang, W.-S. Bao, C. Zhou, M.-S. Jiang, and H.-W. Li, J. Opt. Soc. Amer. B 36, 83 (2019).

[22] C. H. Bennett and G. Brassard, in Proc. IEEE Int. Conf. on Computers, Systems and Signal Processing (IEEE Press, 1984) pp. 175-179.

[23] X. Yuan, Z. Zhang, N. Lütkenhaus, and X. Ma, Phys. Rev. A 94, 062305 (2016).

[24] X. Ma and M. Razavi, Phys. Rev. A 86, 062319 (2012).

[25] M. Lucamarini, Z. L. Yuan, J. F. Dynes, and A. I. Shields, Nature 557, 400 (2018).

[26] X. Ma, P. Zeng, and H. Zhou, Phys. Rev. X 8, 031043 (2018).

[27] M. Tomamichel and A. Leverrier, Quantum 1, 14 (2017).

[28] R. Renner, Security Of QKD, Ph.D. thesis, ETH (2005), Diss. ETH No. 16242, arXiv:quant-ph/0512258.

[29] B. Kraus, N. Gisin, and R. Renner, Phys. Rev. Lett. 95, 080501 (2005).

[30] R. Renner, N. Gisin, and B. Kraus, Phys. Rev. A 72, 012332 (2005).

[31] C.-H. F. Fung, X. Ma, and H. F. Chau, Phys. Rev. A 81, 012318 (2010).

[32] D. Bruß, Phys. Rev. Lett. 81, 3018 (1998).

[33] C. McDiarmid, "On the method of bounded differences," in Surveys In Combinatorics 1989, Lond. Math. Soc. Lect. Notes Series 141, edited by J. Siemons (CUP, 1989) pp. $148-188$.

[34] C. McDiarmid, Combin. Prob. \& Comput. 6, 79 (1997).

[35] W. Hoeffding, J. Amer. Stat. Assoc. 58, 13 (1963). 